\title{
Tumor sólido pseudopapilar de pâncreas: relato de um caso com apresentação incomum
}

\author{
Solid pseudopapillary tumor of the pancreas: report of one uncommon presentation case
}

Felipe Santos Xavier; Cícero de Andrade Urban²; Júlio Cezar Uili Coelho³; Luiz Fernando Bleggi Torres

\section{unitermos}

Neoplasias pancreáticas

Pâncreas

Tumor

Imuno-histoquímica

Diagnóstico

Sistema digestivo

\section{resumo}

Caso clínico atípico de tumor sólido pseudopapilar de pâncreas (TSPPP) acometendo paciente do sexo masculino, 37 anos, com quadro inicial de dor abdominal aguda. Após cirurgia e exame anatomopatológico com imuno-histoquímica, demonstrou padrão condizente com TSPPP e forte positividade para $\beta$-catenina. Dois anos após a cirurgia o paciente encontra-se livre de recidiva. O TSPPP é mais comum em mulheres jovens com quadro clínico de massa ou desconforto abdominal, podendo alguns casos ser assintomáticos. Apresenta crescimento lento e baixo potencial de malignidade, sendo a ressecção cirúrgica o tratamento de escolha com altos índices de cura.

\section{abstract}

Atypical clinical case of solid pseudopapillary tumor (SPPT) of the pancreas in a 37 year-old man with acute abdominal pain. After surgery, anatomopathologic investigation with immunohistochemistry showed pattern in keeping with SPPT and strong positivity for $\beta$-catenin. Two years post-surgery the patient does not present recurrence. The SPPT normally occurs in young women with a history of mass or abdominal discomfort, although in some cases it can be asymptomatic. The tumor has slow growth, low potential for malignancy and surgical resection yields high cure rates.

\section{key words}

Pancreatic neoplasms

Pancreas

Tumor

Immunohistochemistry

Diagnosis

Digestive system

1. Acadêmico de Medicina da Universidade Federal do Paraná (UFPR).

2. Doutor em clínica cirúrgica pelo UFPR; professor titular de Bioética e Metodologia Científica da Universidade Positivo.

3. Pós-doutor pela University of Texas System (UTS; professor titular e coordenador da disciplina de Cirurgia do Aparelho Digestivo da UFPR; chefe dos serviços de Cirurgia do Aparelho Digestivo e Transplante Hepático do Hospital de Clínicas da UFPR.

4. Doutor em Neuropatologia pela Universidade de Londres; professor titular de Patologia Médica da UFPR; pesquisador titular do Instituto Pelé Pequeno Príncipe (IPPP). 


\section{Introdução}

O tumor sólido pseudopapilar de pâncreas (TSPPP) foi descrito pela primeira vez por Frantz, em 1959, com três casos de lesões incomuns de tumor papilar ${ }^{(5)}$. Desde então vários termos têm sido utilizados para designá-lo: neoplasia epitelial papilar, neoplasia cística papilar, neoplasia epitelial papilar e sólida, tumor acinar cístico e sólido, neoplasia sólida e papilar, carcinoma cístico e papilar, tumor papilar cístico e sólido e carcinoma ou tumor pseudopapilar sóli$\mathrm{do}^{(2,3,8)}$. Essa variedade de termos dificultava informações epidemiológicas e a Organização Mundial da Saúde (OMS), em 1996, padronizou como tumor sólido pseudopapilar de pâncreas ${ }^{(9)}$.

\section{Relato de caso}

Paciente masculino, 37 anos, com dor abdominal aguda com menos de 24 horas de duração. Negava outros sintomas. Exames físico e laboratorial normais. Ultrassom (US) de abdome revelou nódulo irregular, sólido, mal delimitado, medindo $5 \mathrm{~cm}$ e localizado no processo uncinado do pâncreas (Figura 1). A ressonância magnética (RM) do abdome confirmou os achados anteriores (Figura 2).

Foi submetido a duodenopancreatectomia com preservação do piloro, sendo extraída massa tumoral de $4,5 \mathrm{~cm}$, rosada, lobulada, homogênea e elástica com áreas amolecidas e friáveis. $\mathrm{O}$ exame microscópico demonstrou neoplasia com padrão de crescimento pseudopapilar, com áreas de infarto e necrose e presença de raros focos de infiltração perineural (Figura 3). O estudo imunohistoquímico (Tabela) confirmou o diagnóstico de TSPPP. O paciente evoluiu com fístula pancreática no $20^{\circ}$ dia do pós-operatório, a qual fechou espontaneamente em 60 dias, e diabetes mellitus tipo 2 (DM2). Está em acompanhamento há dois anos, sem evidência de recidiva.

\section{Discussão}

Os TSPPPs são neoplasias pancreáticas raras e representam cerca de $5 \%$ de todos os tumores císticos do pâncreas ${ }^{(2)}$ e $1 \%$ a $2 \%$ dos tumores exócrinos ${ }^{(1)}$. É mais comum em pacientes na segunda e terceira décadas de vida, com

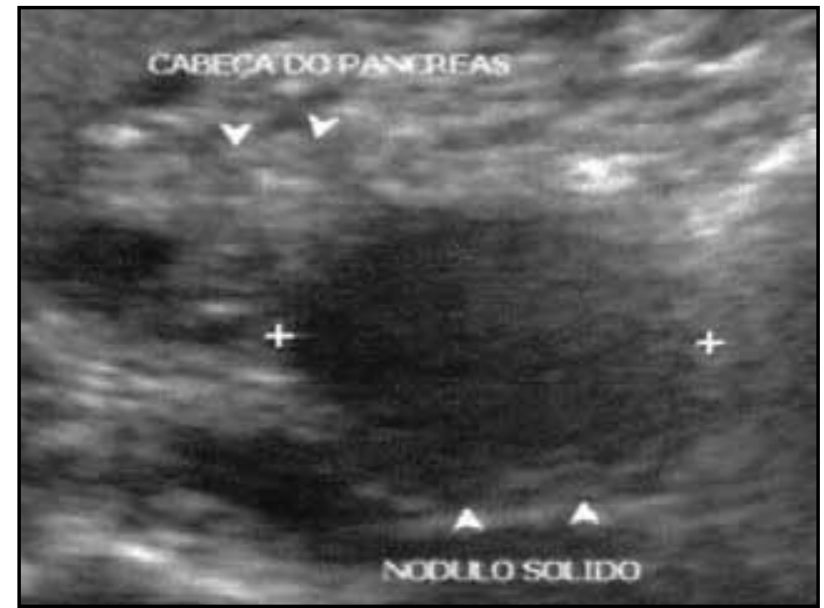

Figura 1 - Ecografia de pâncreas demonstrando TSPPP em topografia de processo uncinado

TSPPP: tumor sólido pseudopapilar de pâncreas.

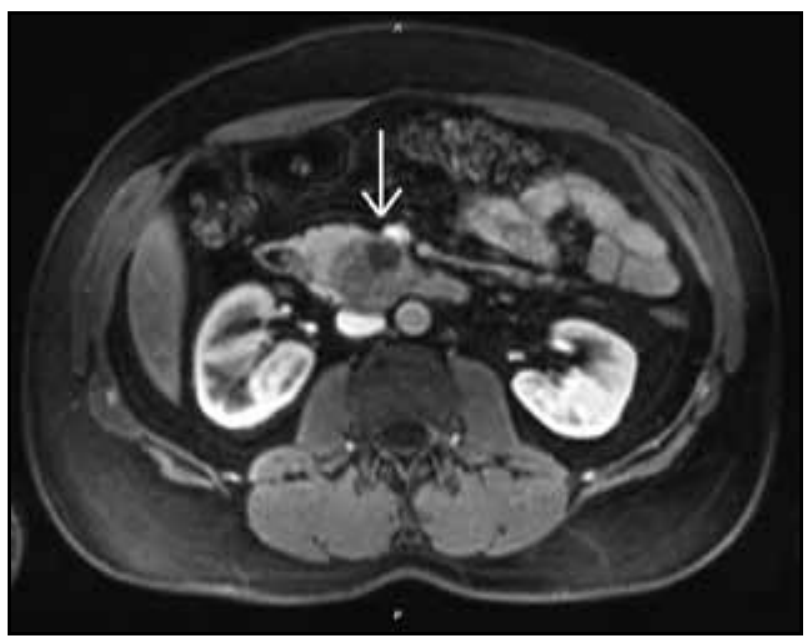

Figura 2 -RM: corte axial ponderado em T1 após contraste mostra lesão sólido-cística no processo uncinado RM: ressonância magnética.

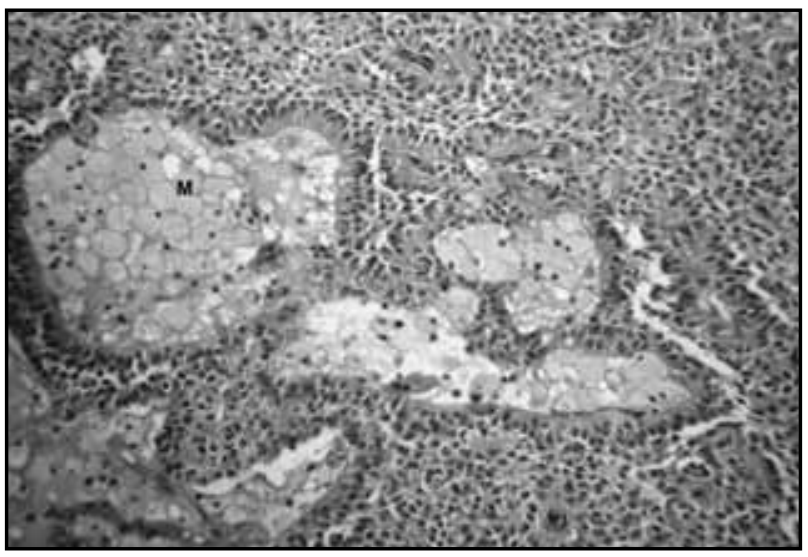

Figura 3A - Micrografia do TSPPP demonstrando áreas de arranjo papilar, sólido e focos de cistificação, rico em macrófago (M) (HE, 100x)

TSPPP: tumor sólido pseudopapilar de pâncreas; HE: hematoxilina e eosina. 


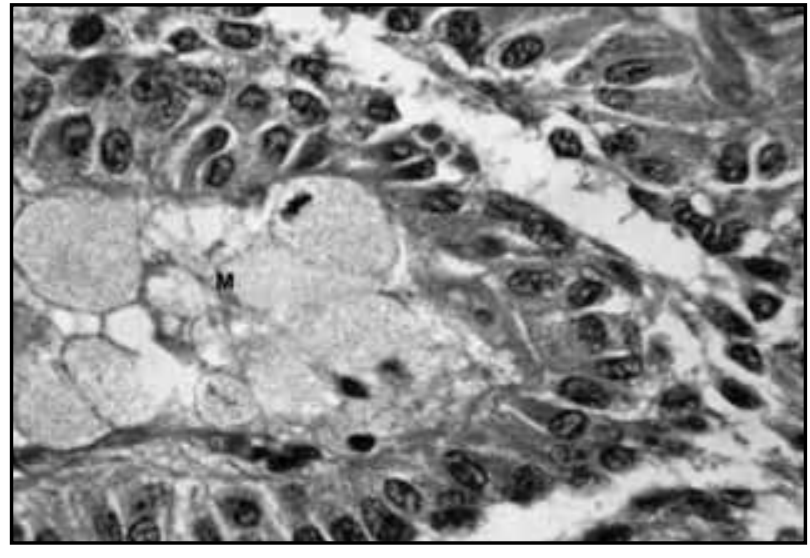

Figura 3B - TSPPP com arranjo papilar contendo macrófagos $(M)$ no eixo conjuntivo central e revestimento por células cilíndricas, citoplasma mediano e núcleos regulares (HE, 400x)

TSPPP: tumor sólido pseudopapilar de pâncreas; HE: hematoxilina e eosina.

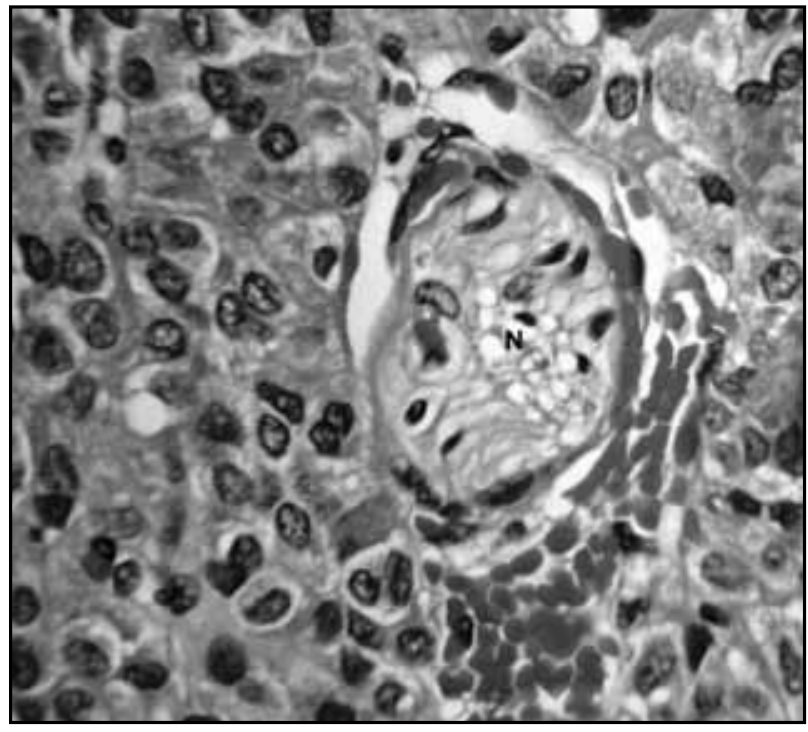

Figura 3C - TSPPP com área focal de invasão perineural (N) (HE, 400x) TSPPP: tumor sólido pseudopapilar de pâncreas; HE: hematoxilina e eosina.

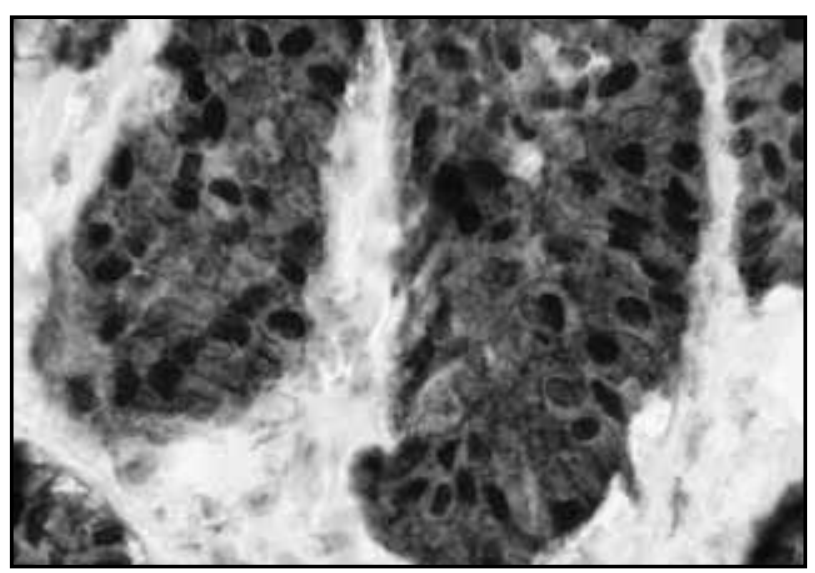

Figura 3D - Imuno-histoquímica para $\beta$-catenina demonstrando intensa reação nuclear e citoplasmática em TSPPP (DAB, 400X) TSPPP: tumor sólido pseudopapilar de pâncreas; DAB: diaminobenzidina.

\begin{tabular}{|c|c|c|}
\hline Tabela & $\begin{array}{l}\text { Resultado do estudo } \\
\text { imuno-histoquímico }\end{array}$ & \\
\hline Anticorpo & Procedência & Resultado \\
\hline$\alpha$-1-antitripsina & $\begin{array}{l}\text { Clone SY38, } \\
\text { Dako }\end{array}$ & Positivo \\
\hline$\beta$-catenina & $\begin{array}{l}\beta \text {-catenin-1, } \\
\text { Dako }\end{array}$ & Positivo \\
\hline AE1AE3 & AE1AE3, Dako & Negativo \\
\hline CD10 & $\begin{array}{c}\text { Clone 56C6, } \\
\text { Novacastra }\end{array}$ & Positivo \\
\hline CD34 & $\begin{array}{l}\text { QEEND-10, } \\
\text { Dako }\end{array}$ & Negativo \\
\hline Cromogranina & RBT, Dako & Negativo \\
\hline Ki67 & $\begin{array}{l}\text { Clone MIB-1, } \\
\text { Dako }\end{array}$ & Positivo \\
\hline Sinaptofisina & RBT, Dako & Positivo \\
\hline Vimentina & Clone V9, Dako & Positivo \\
\hline
\end{tabular}

média de 22 anos $^{(8)}$, ocorrendo na maioria dos casos no sexo feminino ${ }^{(2,3,6-10)}$, contrapondo-se ao presente estudo, com paciente do sexo masculino de 37 anos. Devido a essa predominância no sexo feminino foi proposto que os hormônios sexuais pudessem fazer parte da gênese tumoral, entretanto ensaios imuno-histoquímicos não são unânimes na demonstração de receptores de estrógeno nessas células. Acredita-se que os hormônios sexuais sejam importantes no crescimento, e não na formação, permanecendo a dúvida quanto à origem do tumor ${ }^{(9)}$.

$O$ gene $\beta$-catenina parece estar alterado no TSPPP. A via WNT de sinalização é composta pelos genes polipose adenomatosa do cólon (APC) e $\beta$-catenina, que formam um complexo no interior da célula, levando à destruição da $\beta$-catenina. Em casos com falha na destruição, por sinalização anormal da via ou por alguma mutação, a $\beta$-catenina se acumula no citoplasma, entra no núcleo e se liga ao TCF, um fator de transcrição que ativa diversos genes envolvidos no ciclo celular. Outra função da $\beta$-catenina é se ligar com a E-caderina para manter a adesividade intercelular. Diante disso acredita-se que o acúmulo de $\beta$-catenina em TSPPP possa causar uma desorganização das E-caderinas, sendo responsável pela morfologia sólido-cístico e pseudopapilar desses tumores ${ }^{(1,4)}$. Na imuno-histoquímica com anticorpo anti- $\beta$-catenina e anti-E-caderina há forte reação nuclear e citoplasmática com a $\beta$-catenina e forte reação nuclear com a E-caderina, sugerindo mutação do gene $\beta$-catenina, como observado no presente caso(1). 
O quadro clínico é bastante variado e inespecífico. Durante uma revisão de 718 casos, Papavramidis et al. observaram que a dor abdominal é o sintoma mais comum $(46,5 \%)$, seguido de massa abdominal $(34,84 \%)$, desconforto abdominal (3,89\%), vômitos (3,89\%), náusea $(3,27 \%)$, febre $(1,24 \%)$ e icterícia $(1,09 \%)$, sendo $15,55 \%$ dos pacientes assintomáticos e 3,11\% diagnosticados após um trauma. No caso apresentado o paciente referia dor abdominal aguda com menos de 24 horas de duração e sem outros sintomas associados. Por ser uma neoplasia com baixo grau de malignidade e crescimento lento, é comum a presença de uma massa bastante volumosa ao diagnóstico, oscilando até $34,5 \mathrm{~cm}^{(2,3,7-10)}$, fato discordante em nosso relato, em que o paciente apresentou uma massa de $4,5 \mathrm{~cm}$ por um diagnóstico precoce motivado pelo evento de dor abdominal agu$\mathrm{da}^{(8)}$. As localizações mais comuns são cabeça e cauda do pâncreas, representando $70 \%$ dos tumores, seguidas pelo corpo e, mais raramente, o processo uncinado, que foi a localização encontrada no caso clínico ${ }^{(8)}$. As metástases são incomuns e, quando presentes, o fígado é o órgão mais acometido $(2,7,8)$.

O diagnóstico é realizado por exames de imagem como US, tomografia computadorizada (TC) e $\mathrm{RM}^{(2,8)}$. Recentemente tem sido utilizada a punção aspirativa por agulha fina (PAAF) para um diagnóstico mais acurado em casos difíceis ${ }^{(7,8,10)}$. No caso relatado, a US evidenciou massa sólida e mal delimitada, que foi confirmada com RM, não sendo realizada PAAF devido à suspeita de adenocarcinoma. O diagnóstico diferencial inclui cistos congênitos, pseudocistos, cistoadenomas serosos, tumor cístico mucinoso, adenocarcinoma e tumores metastáticos ${ }^{(2,7)}$.

A avaliação anatomopatológica varia conforme o tamanho do tumor. Lesões pequenas são menos propensas a cistificações, tendo cápsula delgada e quantidade variável de fibrose. Já as lesões maiores possuem cápsula fibrosa mais espessa e combinações variáveis de áreas hemorrágicas e necróticas ${ }^{(2,8)}$. A microscopia revela um padrão sólido pseudopapilar com microcirculação abundante. O crescimento celular é uniforme, com citoplasma levemente eosinofílico, ao redor de um eixo fibroconjuntivo mixomatoso e presença de raras mitoses ${ }^{(2,8,9)}$. O TSPPP possui um padrão imuno-histoquímico característico $^{(2)}$. As células neoplásicas são positivas para vimentina em todos os tumores e frequentemente positivas para $\alpha$-1-antitripsina e $\alpha-1$-antiquimiotripsina. Alguns casos apresentam reação para enolase neuroespecífica, sinaptofisina, citoqueratina, proteína $S-100$ e receptores de hormônios sexuais ${ }_{(2,8)}$. Nos últimos anos tem sido relatada forte positividade para CD10 e CD56 (1). Nosso caso apresentou perfil imuno-histoquímico similar ao descrito na literatura.

O tipo de tratamento cirúrgico depende da localização do tumor e objetiva a preservação máxima de estruturas adjacentes. A pancreatectomia distal com ou sem preservação esplênica pode ser realizada para tumores localizados no corpo e na cauda do pâncreas. A duodenopancreatectomia é indicada quando a lesão se localiza na cabeça do pâncreas. A enucleação é uma opção em tumores de pequeno tamanho e bem localizados, principalmente em crianças ${ }^{(2,3,8)}$. Linfadenectomia regional não está indicada, exceto se houver presença de linfonodos volumosos com suspeita de invasão(2, 3, 8). Metástases, principalmente as hepáticas, não são critérios de exclusão do tratamento cirúrgico, e a ressecção pode oferecer um controle da doença ou mesmo a cura ${ }^{(2,3,8)}$. A experiência com tratamentos adjuvantes é limitada, e os altos índices de cura obtidos com a cirurgia tornam a quimioterapia e a radioterapia pouco indicadas. Há relatos na literatura sobre lesões irressecáveis tratadas com radioterapia, obtendo alívio dos sintomas, controle da doença e melhora da qualidade de vida ${ }^{(9,11)}$.

O prognóstico da doença é excelente, mesmo quando apresenta metástase a distância. Estima-se que mais de $95 \%$ dos pacientes com doença localizada são curados com a ressecção cirúrgica, e a sobrevida dos pacientes com metástase hepática normalmente ultrapassa cinco anos.

\section{Agradecimentos}

Os autores agradecem à Farmacêutica Flávia Marder Torres pelas reações imuno-histoquímicas e à Dra. Fabíola Medeiros, da Mayo Clinic, EUA, por imuno-histoquímica complementar e revisão diagnóstica. 


\section{Referências}

1. CHETTY, R.; SERRA, S. Membrane loss and aberrant nuclear localization of E-cadherin are consistent features of solid pseudopapillary tumor of the pancreas. An imunohistochemical study using two antibodies recognizing different domains of the E-cadherin molecule. Histopathology, v. 52, p. 325-30, 2008.

2. COSTA, S. R. P. et al. Tumor papilar sólido-cístico do pâncreas: aspectos clínico-radiológicos e resultados cirúrgicos em cinco pacientes operados. Einstein, v. 5, n. 2, p. 161-5, 2007.

3. COSTA-NETO, G. D. et al. Tumor sólido-cístico pseudopapilar do pâncreas (tumor de Frantz). Estudo de quatro casos. Arq Gastroenterol, v. 41, n. 4, p. 259-62, 2004.

4. EL-BAHRAWY, M. A. et al. E-cadherin/catenin complex status in solid pseudopapillary tumor of the pancreas. Am J Surg Pathol, v. 32, n. 1, p. 1-7, 2008.

5. FRANTZ, V. K. Tumors of the Pancreas. Anonymous Atlas of Tumor Pathology. Armed Forces Institute of Pathology, v. 7, n. 27-8, p. 32-3, 1959.
6. GEERS, C. et al. Solid and pseudopapillary tumor of the pancreas-review and new insights into pathogenesis. Am J Surg Pathol, v. 30, n. 10, p.1243-9, 2006.

7. MACEDO, T. A. et al. Tumor de Frantz: relato de um caso. Rev Bras Cancerologia, v. 50, n. 1, p. 33-5, 2004.

8. PAPAVRAMIDIS, T.; PAPAVRAMIDIS, S. Solid pseudopapillary tumors of the pancreas: review of 718 patients reported in English literature. J Am Coll Surg, v. 200, n. 6, p. 965-72, 2005.

9. PINTO JR, F. E. L. et al. Neoplasia papilar cística do pâncreas. Rev Col Bras Cir, v. 28, n. 3, p. 304-6, 2001.

10. VINCENT, P. J. et al. Gruber Frantz tumor. Medical J Armed Forces India, v. 59, n. 4, p. 355-7, 2003.

11. ZAULS, J. A. et al. Intensity-modulated radiation therapy for unresectable solid pseudopapillary tumor of the pancreas. Am J Clin Oncol, v. 29, n. 6, p. 639-40, 2006. 